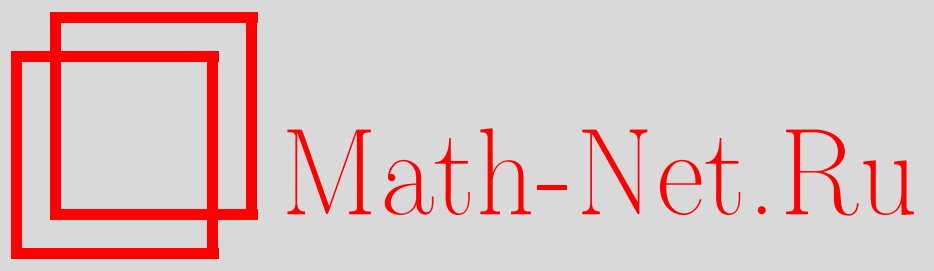

М. В. Поляков, А. А. Владимиров, Главные инфракрасные логарифмы для сигма-модели с полями на произвольном римановом многообразии, ТМ $\Phi, 2011$, том 169, номер 1, 158-166

DOI: https://doi.org/10.4213/tmf6717

Использование Общероссийского математического портала Math-Net.Ru подразумевает, что вы прочитали и согласны с пользовательским соглашением http://www . mathnet.ru/rus/agreement

Параметры загрузки:

IP : 3.89.197.203

26 апреля 2023 г., 09:08:59

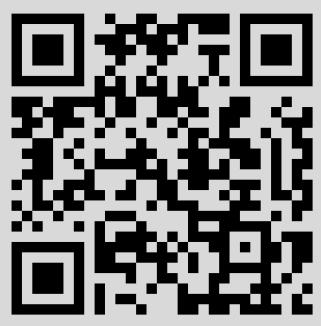




\section{ГЛАВНЫЕ ИНФРАКРАСНЫЕ ЛОГАРИФМЫ ДЛЯ СИГМА-МОДЕЛИ С ПОЛЯМИ НА ПРОИЗВОЛЬНОМ РИМАНОВОМ МНОГООБРАЗИИ}

Получено нелинейное рекуррентное уравнение для инфракрасных главных логарифмов в четырехмерной сигма-модели с полями на произвольном римановом многообразии. Полученное уравнение позволяет вычислить главные инфракрасные логарифмы в сущности до неограниченного петлевого порядка в терминах геометрических характеристик риманова многообразия. Решение для $S U(\infty)$-модели главного кирального поля в произвольном числе размерностей в приближении главных логарифмов редуцируется к решению очень простого рекуррентного уравнения. Этот результат прокладывает путь к решению модели в произвольном числе размерностей при $N \rightarrow \infty$.

Ключевые слова: ренормализационнная группа, сигма-модели, большие $N$.

Памяти Александра Николаевича Васильева

\section{1. ВВЕДЕНИЕ}

Эффективные теории поля представляют собой неперенормируемые теории поля, позволяющие исследовать инфракрасное (низкоэнергетическое) поведение различных физических систем (см. разнообразные примеры в работах [1], [2]). Стандартным приемом исследования асимптотического поведения перенормируемых теорий поля является метод уравнений ренормализационной группы (УРГ). Для эффективных теорий поля метод УРГ следует модифицировать, поскольку число контрчленов быстро возрастает с ростом петлевого порядка. Тонкости перенормировки в квантовой теории поля описаны в замечательной книге А. Н. Васильева [1].

Возможность систематического построения УРГ для неперенормируемых квантовых теорий поля была продемонстрирована в работе [3]. В частности, было показано, что ряд главных логарифмов (ГЛ) можно получить путем вычисления однопетлевых диаграмм. Однако решение УРГ, полученное в работе [3], требует вычисления нетривиальных однопетлевых диаграмм. Число диаграмм быстро возрастает с ростом числа петель, поэтому использование этого метода на практике - непростая

*Петербургский институт ядерной физики, Гатчина, Ленинградская обл., Россия. E-mail: maxime.poliakoff@googlemail.com

${ }^{\dagger}$ Institut für Theoretische Physik II, Ruhr-Universität, Bochum, Germany 
задача. Метод, предложенный в работе [3], был применен в работах [4] к вычислению ГЛ с точностью до пяти петель для постоянной распада и массы пиона и к вычислению с точностью до четырех петель в мезон-мезонном рассеянии в массивной $O(N+1) / O(N)$ сигма-модели. Авторы работы [5], используя дисперсионные методы, вычислили ГЛ с точностью до трех петель для $(\pi \pi)$-рассеяния в безмассовой киральной теории возмущений. Недавно в работах [6]-[8] был развит совершенно другой метод вычисления ГЛ для широкого класса неперенормируемых безмассовых теорий поля. Нелинейные рекуррентные уравнения, выведенные в работах [6]-[8], позволяют получить вклады ГЛ до практически неограниченного петлевого порядка без выполнения нетривиальных петлевых вычислений для каждого петлевого порядка.

В настоящей работе мы вычисляем вклад инфракрасных ГЛ для четырехмерной сигма-модели с полями на произвольном римановом многообразии. Одна из наших основных задач состоит в интерпретации ГЛ в терминах геометрических характеристик риманова многообразия. В частности, такой подход позволяет редуцировать решение для $S U(\infty)$-модели главного кирального поля при произвольном числе размерностей в приближении ГЛ к решению очень простого рекуррентного уравнения. Этот результат прокладывает путь к решению модели для произвольного числа размерностей при $N \rightarrow \infty$.

Работа построена следующим образом. Самые общие основные результаты приведены в уравнениях (5), (6) раздела 2. В этом разделе также приведены, по-видимому впервые, двухпетлевые результаты для общей сигма-модели (см. формулы (10)). В разделе 3 показано, как работает общий метод раздела 2, на "типовом примере" $N$-мерной сферы в качестве таргет-пространства. Наиболее интересные и нетривиальные результаты для $S U(\infty)$-модели главного кирального поля - это уравнения (13) и (14) из раздела 4.

\section{2. СИГМА-МОДЕЛЬ С ПОЛЯМИ НА ПРОИЗВОЛЬНОМ РИМАНОВОМ МНОГООБРАЗИИ}

Рассмотрим наиболее общую сигма-модель с полями на произвольном римановом многообразии. Для двух пространственно-временных измерений соответствующая модель является перенормируемой [9], а инфракрасная асимптотика ГЛ определяется однопетлевым УРГ, которое является не чем иным, как уравнением потока Риччи для метрики. В случае более высоких пространственно-временных измерений вычисление ГЛ является существенно нетривиальной задачей. Насколько известно авторам, для общей сигма-модели были вычислены только однопетлевые ГЛ в размерности четыре [10], [11]. В настоящей работе мы покажем, как вычислить ГЛ для произвольного петлевого порядка в общей сигма-модели для четырех пространственно-временных измерений ${ }^{1)}$.

Действие имеет вид

$$
S=\int d^{4} x \frac{1}{2} g_{a b}(\phi) \partial_{\mu} \phi^{a} \partial_{\mu} \phi^{b}
$$

1) Обобщение на случай $D \geqslant 4$ является простым, и его можно выполнить, используя метод, предложенный в работе [8]. 
где $g_{a b}(\phi)$ - метрика на римановом многообразии. Без потери общности можно рассмотреть компактное риманово многообразие с положительной сигнатурой метрики. Используя произвол выбора координат $\left(\phi^{a}\right)$ на римановом многообразии, мы фиксируем метрику так, что $g_{a b}(0)=\delta_{a b}$ и $g_{a b}(\phi) \phi^{b}=\delta_{a b} \phi^{b}$. Последнее условие соответствует выбору нормальных координат на римановом многообразии [12]. В этих координатах геодезические в окрестности $\phi^{a}=0$ представляют собой просто прямые линии, а метрика разлагается вблизи $\phi^{a}=0$ следующим образом:

$$
g_{a b}(\phi)=\delta_{a b}-\frac{1}{3} \stackrel{\circ}{R}_{a c b d} \phi^{c} \phi^{d}+O\left(\phi^{3}\right),
$$

где $\stackrel{\circ}{R} a c b d-$ риманов тензор кривизны в точке $\phi^{a}=0$. Подставляя разложение $(2)$ в действие (1), легко вычислить амплитуду рассеяния $\phi^{a}+\phi^{b} \rightarrow \phi^{c}+\phi^{d}$ на древесном уровне:

$$
A_{a b c d}^{\mathrm{tree}}(s, \cos \theta)=\frac{s}{2}\left(-\left[\stackrel{\circ}{R}_{a c b d}+\stackrel{\circ}{R}_{a d b c}\right] P_{0}(\cos \theta)+\stackrel{\circ}{R}_{a b c d} P_{1}(\cos \theta)\right),
$$

где $s$ - переменная Мандельштама, $\theta$ - угол рассеяния центра масс, а $P_{l}$ - многочлены Лежандра. Это выражение дает главную инфракрасную асимптотику амплитуды рассеяния. Поправки к полученному результату возникают из петлевых вкладов. Простые соображения, основанные на подсчете степеней, показывают, что $(n-1)$-петлевой вклад имеет вид вклада ГЛ $s\left[s \ln \left(\mu^{2} / s\right)\right]^{n-1}$. Для $l$-й парциальной волновой амплитуды, определенной как

$$
t_{a b c d}^{l}(s)=\frac{1}{64 \pi} \int_{0}^{\pi} d \theta \sin \theta A_{a b c d}(s, \cos \theta) P_{l}(\cos \theta),
$$

можно записать общий вид разложения ГЛ:

$$
t_{a b c d}^{l}(s)=\frac{\pi}{2} \frac{1}{2 l+1} \sum_{n=1}^{\infty}\left(\omega_{n l}\right)_{a b c d}\left(\frac{s}{16 \pi^{2}}\right)^{n} \ln ^{n-1}\left(\frac{\mu^{2}}{s}\right)+O(\mathrm{NLL}),
$$

где $O(\mathrm{NLL})$ означает поправки к главному логарифмическому приближению. Здесь $\left(\omega_{n l}\right)_{a b c d}-$ коэффициенты ряда ГЛ, которые зависят только от геометрических характеристик таргет-пространства. Эти коэффициенты отличны от нуля только при $l \leqslant n$, где $n-1$ - число петель. На древесном уровне амплитуда (3) соответствует $n=1$, и в этом случае коэффициенты отличны от нуля только при $l=0$ и $l=1$ :

$$
\left(\omega_{10}\right)_{a b c d}=-\frac{1}{2}\left(\stackrel{\circ}{R}_{a c b d}+\stackrel{\circ}{R}_{a d b c}\right), \quad\left(\omega_{11}\right)_{a b c d}=\frac{1}{2} \stackrel{\circ}{R}_{a b c d} .
$$

Теперь можно использовать предложенный в работе [8] общий метод получения рекуррентного уравнения, который позволяет выразить высшие петлевые коэффициенты ряда ГЛ в терминах древесных коэффициентов (5). Этот метод основан на общих принципах квантовой теории поля - аналитичности, унитарности и кроссинг-симметрии; его описание можно найти в [8], в настоящей работе мы приводим окончательный результат для рекуррентного уравнения:

$$
\begin{aligned}
& \left(\omega_{n l}\right)_{a b c d}=\frac{1}{2(n-1)}\left[\sum_{i=1}^{n-1} \frac{\left(\omega_{i l}\right)_{a b \alpha \beta}\left(\omega_{n-i, l}\right)_{c d}^{\beta \alpha}}{2 l+1}+\right. \\
& \left.+\sum_{i=1}^{n-1} \sum_{l^{\prime}=0}^{n-1} \frac{\left(\omega_{i l^{\prime}}\right)_{a d \alpha \beta}\left(\omega_{n-i, l^{\prime}}\right)_{c b}^{\beta \alpha} \Omega_{n}^{l^{\prime} l}+(-1)^{l+l^{\prime}}\left(\omega_{i l^{\prime}}\right)_{a c \alpha \beta}\left(\omega_{n-i, l^{\prime}}\right)_{b d}^{\beta \alpha} \Omega_{n}^{l^{\prime} l}}{2 l^{\prime}+1}\right] .
\end{aligned}
$$


Здесь $\Omega_{n}^{l^{\prime} l}-$ кроссинг-матрица в пространстве момента импульса, которая имеет вид

$$
\Omega_{n}^{l^{\prime} l}=\frac{2 l+1}{2^{n+1}} \int_{-1}^{1} d z P_{l^{\prime}}\left(\frac{z+3}{z-1}\right) P_{l}(z)(z-1)^{n} .
$$

Уравнение (6) выражает коэффициенты ряда ГЛ для высших петель в терминах низших петель. Начальная точка для рекурсии дается выражениями на древесном уровне (5).

Легко проверить, что решение уравнения (6) удовлетворяет симметриям

$$
\left(\omega_{n l}\right)_{a b c d}=(-1)^{l}\left(\omega_{n l}\right)_{b a c d}=(-1)^{l}\left(\omega_{n l}\right)_{a b d c}=\left(\omega_{n l}\right)_{c d a b}
$$

которые на самом деле являются следствием бозе-симметрии и сохранения четности. Кроме того, уравнение (6) обладает симметриями

$$
\left(\sum_{l^{\prime}=0}^{n} \omega_{n l^{\prime}} \Omega_{n}^{l^{\prime} l}\right)_{a b c d}=\left(\omega_{n l}\right)_{a d c b}, \quad\left(\sum_{l^{\prime}=0}^{n} \omega_{n l^{\prime}}(-1)^{l+l^{\prime}} \Omega_{n}^{l^{\prime} l}\right)_{a b c d}=\left(\omega_{n l}\right)_{a c b d}
$$

которые могут быть весьма полезны при получении точного решения рекуррентного уравнения (6).

Мы пока не нашли аналитического решения уравнения (6), поэтому приведем здесь выражения для коэффициентов ряда ГЛ в двух петлях. Однопетлевые коэффициенты в терминах геометрических характеристик имеют следующий вид:

$$
\begin{aligned}
& \left(\omega_{20}\right)_{a b c d}=\frac{1}{4}\left[R_{a \beta_{1} b \beta_{2}} R_{c}^{\beta_{1} \beta_{2}}+R_{a \beta_{1} b \beta_{2}} R_{c d}^{\beta_{2} \beta_{1}}\right]+ \\
& +\frac{1}{6}\left[R_{a \beta_{1} c \beta_{2}} R_{b}{ }_{d}^{\beta_{1} \beta_{2}}+R_{a \beta_{1} d \beta_{2}} R_{b}{ }_{c}^{\beta_{1} \beta_{2}}\right]-\frac{5}{72}\left[R_{a c \beta_{1} \beta_{2}} R^{\beta_{1} \beta_{2}}{ }_{b d}+R_{a d \beta_{1} \beta_{2}} R^{\beta_{1} \beta_{2}} b c\right], \\
& \left(\omega_{21}\right)_{a b c d}=-\frac{1}{24} R_{a b \beta_{1} \beta_{2}} R^{\beta_{1} \beta_{2}}{ }_{c d}+ \\
& +\frac{1}{4}\left[R_{a \beta_{1} c \beta_{2}} R_{b}^{\beta_{1} \beta_{2}}-R_{a \beta_{1} d \beta_{2}} R_{b}^{\beta_{1} \beta_{2}}\right]-\frac{1}{12}\left[R_{a c \beta_{1} \beta_{2}} R^{\beta_{1} \beta_{2}}{ }_{b d}-R_{a d \beta_{1} \beta_{2}} R^{\beta_{1} \beta_{2}} b c\right], \\
& \left(\omega_{22}\right)_{a b c d}=\frac{1}{12}\left[R_{a \beta_{1} c \beta_{2}} R_{b}^{\beta_{1} \beta_{2}}+R_{a \beta_{1} d \beta_{2}} R_{b c}^{\beta_{1} \beta_{2}}\right]-\frac{1}{72}\left[R_{a c \beta_{1} \beta_{2}} R^{\beta_{1} \beta_{2}} b d+R_{a d \beta_{1} \beta_{2}} R^{\beta_{1} \beta_{2}} b c\right] .
\end{aligned}
$$

Здесь и ниже тензоры Римана берутся в точке $\phi^{a}=0$. Двухпетлевой результат записывается как

$$
\begin{aligned}
\left(\omega_{30}\right)_{a b c d} & =\chi_{a b c d}-\frac{1}{2} \psi_{a b c d}+\frac{1}{3} \psi_{a d b c}-\frac{1}{4} \chi_{a d b c} \\
\left(\omega_{31}\right)_{a b c d} & =\frac{1}{2} \psi_{a b c d}-\frac{1}{2} \psi_{a d b c}-\frac{9}{20} \chi_{a d b c} \\
\left(\omega_{32}\right)_{a b c d} & =\frac{1}{6} \psi_{a d b c}-\frac{1}{4} \chi_{a d b c} \\
\left(\omega_{33}\right)_{a b c d} & =\frac{1}{20} \chi_{a d b c}
\end{aligned}
$$


где

$$
\begin{aligned}
& \chi_{a b c d}=\left[R_{a \beta_{1} b \beta_{2}}\left(-\frac{1}{72} R_{c \beta_{3} \beta_{4}}^{\beta_{1}} R_{d}^{\beta_{3} \beta_{2} \beta_{4}}-\frac{7}{144} R_{c \beta_{3} \beta_{4}} R_{d}^{\beta_{4} \beta_{2} \beta_{3}}-\frac{1}{48} R_{c \beta_{3} \beta_{4}}^{\beta_{2}} R_{d}^{\beta_{4} \beta_{1} \beta_{3}}\right)+\right. \\
& +(a \rightarrow b \rightarrow d \rightarrow c \circlearrowleft)+\frac{1}{144} R_{a \beta_{1} b \beta_{2}}\left(R_{c \beta_{3} d \beta_{4}}-R_{c \beta_{4} d \beta_{3}}\right) R^{\beta_{1} \beta_{2} \beta_{3} \beta_{4}}- \\
& \left.-\frac{1}{8} R_{a \beta_{1} b \beta_{2}}\left(R_{c \beta_{3} d \beta_{4}}+R_{c \beta_{4} d \beta_{2}}\right) R^{\beta_{1} \beta_{3} \beta_{2} \beta_{4}}\right]+(c \leftrightarrow b), \\
& \psi_{a b c d}=\left[R_{a b \beta_{1} \beta_{2}}\left(-\frac{1}{72} R_{c \beta_{3} \beta_{4}}^{\beta_{1}} R_{d}^{\beta_{3} \beta_{2} \beta_{4}}-\frac{1}{36} R_{c \beta_{3} \beta_{4}} R_{d}^{\beta_{4} \beta_{2} \beta_{3}}\right)+\right. \\
& \left.+\left(\begin{array}{l}
a \leftrightarrow c \\
b \leftrightarrow d
\end{array}\right)-(b \leftrightarrow c)-(a \rightarrow b \rightarrow d \rightarrow c \circlearrowleft)\right]+ \\
& +\left[R _ { b \beta _ { 1 } d \beta _ { 2 } } \left(\frac{1}{36} R_{a \beta_{3} \beta_{4}}^{\beta_{2}} R_{c}^{\beta_{3} \beta_{1} \beta_{4}}+\frac{1}{72} R_{a \beta_{3} \beta_{4}}^{\beta_{1}} R_{c}^{\beta_{3} \beta_{2} \beta_{4}}+\right.\right. \\
& \left.\left.+\frac{17}{144} R_{a \beta_{3} \beta_{4}}^{\beta_{2}} R_{c}^{\beta_{4} \beta_{2} \beta_{3}}+\frac{13}{144} R_{a \beta_{3} \beta_{4}}^{\beta_{1}} R_{c}^{\beta_{4} \beta_{2} \beta_{3}}\right)+\left(\begin{array}{l}
a \leftrightarrow b \\
c \leftrightarrow d
\end{array}\right)\right]+ \\
& +\frac{3}{16} R_{a \beta_{1} c \beta_{2}} R_{b \beta_{3} d \beta_{4}} R^{\beta_{1} \beta_{3} \beta_{2} \beta_{4}}+ \\
& +\left(\frac{1}{144} R_{a b \beta_{1} \beta_{2}} R_{c d \beta_{3} \beta_{4}}-\frac{1}{72} R_{a \beta_{1} c \beta_{2}} R_{b \beta_{3} d \beta_{4}}\right) R^{\beta_{1} \beta_{2} \beta_{3} \beta_{4}}
\end{aligned}
$$

Выражения для высших петель легко получить путем дальнейших итераций уравнения (6). Мы их не приводим, поскольку они являются довольно громоздкими. Вместо этого ниже мы обсуждаем частные случаи римановых многообразий: $N$-мерную сферу $S^{N}$ и групповое многообразие, соответствующее простой группе Ли $G$. Последний случай представляет собой сигма-модель главного кирального поля, имеющую много общего с теорией Янга-Миллса.

\section{3. СИГМА-МОДЕЛЬ С ПОЛЯМИ НА СФЕРЕ $S^{N}$}

Метрика на $N$-мерной сфере $S^{N}$ в нормальных координатах имеет следующий вид:

$$
g_{a b}(\phi)=F^{2}\left(\delta_{a b} \frac{\sin ^{2}(|\phi| / F)}{|\phi|^{2}}+\frac{\phi_{a} \phi_{b}}{|\phi|^{2}}\left[\frac{1}{F^{2}}-\frac{\sin ^{2}(|\phi| / F)}{|\phi|^{2}}\right]\right),
$$

где $|\phi| \equiv \sqrt{\phi_{a} \phi^{a}}$ и $F$ - радиус сферы $S^{N}$. Для теории (1) в четырех пространственно-временных измерениях постоянная $F$ имеет размерность массы, а в случае $S^{3}$ соответствует постоянной распада пиона эффективного кирального лагранжиана для КХД с двумя ароматами. Тензор Римана, тензор Риччи и скалярная кривизна сферы $S^{N}$ имеют вид

$$
R_{a b c d}=\frac{1}{F^{2}}\left(g_{a c} g_{b d}-g_{a d} g_{b c}\right), \quad R_{a b}=\frac{N-1}{F^{2}} g_{a b}, \quad R=\frac{N(N-1)}{F^{2}} .
$$


Подставляя эти выражения в общие решения (9), (10), получаем однопетлевые коэффициенты ряда ГЛ:

$$
\begin{aligned}
\left(\omega_{20}\right)_{a b c d} & =\frac{1}{18 F^{4}}\left[(3 N-1)\left(\delta_{a d} \delta_{b c}+\delta_{a c} \delta_{b d}\right)+(9 N-17) \delta_{a b} \delta_{c d}\right], \\
\left(\omega_{21}\right)_{a b c d} & =\frac{1}{4 F^{4}}(N-3)\left(\delta_{a d} \delta_{b c}-\delta_{a c} \delta_{b d}\right), \\
\left(\omega_{22}\right)_{a b c d} & =\frac{1}{36 F^{4}}\left[(3 N-5)\left(\delta_{a d} \delta_{b c}+\delta_{a c} \delta_{b d}\right)+4 \delta_{a b} \delta_{c d}\right] .
\end{aligned}
$$

Двухпетлевые коэффициенты имеют следующий вид:

$$
\begin{array}{ll}
\left(\omega_{3 l}\right)_{a b c d}=\frac{1}{288 F^{6}}\left[a_{3 l}\left(\delta_{a d} \delta_{b c}+\delta_{a c} \delta_{b d}\right)+b_{3 l} \delta_{a b} \delta_{c d}\right], & l=0,2, \\
\left(\omega_{3 l}\right)_{a b c d}=\frac{1}{1440 F^{6}} c_{3 l}\left(\delta_{a d} \delta_{b c}-\delta_{a c} \delta_{b d}\right), & l=1,3,
\end{array}
$$

где

$$
\begin{array}{ll}
a_{30}=-59+27 N-18 N^{2}, & b_{30}=86-158 N+72 N^{2}, \\
a_{32}=-39+47 N-18 N^{2}, & b_{32}=10+10 N, \\
c_{31}=441-333 N+162 N^{2}, & c_{33}=49-37 N+18 N^{2} .
\end{array}
$$

В приведенных выше формулах для первых двух петель можно заметить закономерность, которой подчиняются главные коэффициенты при больших $N$. Действительно, при больших $N$ легко получить ${ }^{2)}$ решение уравнения (6) в случае сферы $S^{N}$ [6], [8]:

$$
\left(\omega_{n l}\right)_{a b c d}^{\text {Large } N}=\frac{1}{F^{2}}\left(-\frac{N}{2 F^{2}}\right)^{n-1}\left[\frac{(2 l+1) n n !}{(n+l+1) !}\left(\delta_{a d} \delta_{b c}+(-1)^{l} \delta_{a c} \delta_{b d}\right)+(-1)^{n-1} \delta_{l 0} \delta_{a b} \delta_{c d}\right] .
$$

Как можно видеть, сигма-модель с полями на $S^{N}$ можно решить в пределе больших $N$ для произвольного числа пространственно-временных измерений. Это является хорошо известным результатом. Рекордные вычисления $1 / N$-поправок были представлены в работе [13], где они получены очень элегантным методом конформного бутстрапа. Из решения при больших $N$ (12) мы видим, что после суммирования амплитуда ГЛ имеет полюс при $l=0$ и синглетный по $O(N)$ канал. Этот полюс соответствует вкладу вспомогательного скалярного поля, которое мы ввели для решения сигма-модели на сфере $S^{N}$ в пределе больших $N$.

Заметим, что все результаты, полученные в настоящем разделе для $S^{N}$, справедливы также для любого простого связного конформного плоского риманова многообразия.

\footnotetext{
${ }^{2)}$ Мы использовали изометрии $S^{N}$-многообразия (см. раздел 4$)$.
} 


\section{4. ГЛАВНОЕ КИРАЛЬНОЕ ПОЛЕ: СПЕЦИАЛЬНЫЙ СЛУЧАЙ $S U(\infty)$}

Важным является случай сигма-модели с таргет-пространством $(G \times G) / G$, где $G$ - простая группа Ли. Это модель главного кирального поля. Метрика в нормальных координатах на $(G \times G) / G$ имеет вид

$$
g_{a b}(\phi)=\int_{0}^{1} d \alpha(1-\alpha) \operatorname{tr}\left\{\exp \left(i \alpha \frac{t^{c} \phi^{c}}{F}\right) t^{a} \exp \left(-i \alpha \frac{t^{c} \phi^{c}}{F}\right) t^{b}\right\},
$$

где $t^{a}$ - генераторы группы $G$ в фундаментальном представлении, нормированные условием $\operatorname{tr}\left(t^{a} t^{b}\right)=2 \delta^{a b}, F$ - параметр массовой размерности единица. Для группы $G=S U(N)$ этот параметр соответствует постоянной распада бозона Намбу-Голдстоуна в киральном лагранжиане для $\mathrm{KXД} \mathrm{с} N$ безмассовыми кварковыми ароматами. Ниже мы рассмотрим случай $G=S U(N)$. Во многих отношениях $S U(N)$-модель главного кирального поля аналогична теории Янга-Миллса, например ее предел при больших $N$ соответствует суммированию планарных диаграмм. Вопреки многочисленным надеждам [14] эта модель не решается в пределе больших $N$ при произвольном числе пространственно-временных измерений. Мы обсудим этот предел ниже в рамках приближения ГЛ.

Тензор Римана, тензор Риччи, а также скалярная кривизна для многообразия $S U(N) \times S U(N) / S U(N)$ имеют следующий вид:

$$
R_{a b c d}=\frac{1}{8 F^{2}} \operatorname{tr}\left(\left[t^{a}, t^{b}\right]\left[t^{c}, t^{d}\right]\right)+O\left(\phi^{2}\right), \quad R_{a b}=\frac{N}{F^{2}} g_{a b}, \quad R=\frac{N\left(N^{2}-1\right)}{F^{2}} .
$$

В принципе, можно использовать эти выражения для наших основных уравнений (6), (5), чтобы получить коэффициенты ряда ГЛ. Однако результаты, особенно для высших петлевых порядков, оказываются довольно громоздкими, поэтому здесь разумно использовать изометрии многообразия $S U(N) \times S U(N) / S U(N)$. Для амплитуды (4) изометрии позволяют разложить ее по проекторам на неприводимые представления группы $S U(N)$ :

$$
t_{a b c d}^{l}(s)=\sum_{R=1}^{7} P_{a b c d}^{R} t_{R}^{l}(s),
$$

где $P_{a b c d}^{R}$ - проекторы на неприводимые представления группы $S U(N)$, которые появляются в произведении $\mathrm{Adj} \times \mathrm{Adj}$. Вообще говоря, имеются семь представлений с размерностями ${ }^{3)}$

$$
\begin{aligned}
d_{R}= & \left(1, N^{2}-1, \frac{N^{2}(N+1)(N-3)}{4}, \frac{N^{2}(N-1)(N+3)}{4},\right. \\
& \left.N^{2}-1, \frac{\left(N^{2}-4\right)\left(N^{2}-1\right)}{4}, \frac{\left(N^{2}-4\right)\left(N^{2}-1\right)}{4}\right) .
\end{aligned}
$$

Явные выражения для проекторов $P_{a b c d}^{R}$ выведены в работе [15]. Заметим, что представления с числами $R=2$ и $R=5$ соответствуют симметричному и антисимметричному присоединенным представлениям группы $S U(N)$. В работе [15] было

\footnotetext{
3) Для читателя, знакомого с теорией группы $S U(3)$, это $d_{R}=\left(1,8_{S},-, 27,8_{A}, 10, \overline{10}\right)$.
} 
показано, что в пределе больших $N$ остаются только эти два присоединенных представления, а вклад других представлений является поправкой порядка $1 / N$. Это очень интересное наблюдение, потому что оно позволяет существенно уменьшить число геометрических объектов в пределе больших $N$.

Решение для коэффициентов ряда ГЛ в произвольном петлевом порядке при $N \rightarrow \infty$ в случае $S U(N)$-модели главного кирального поля имеет вид

$$
\left(\omega_{n l}\right)_{\text {abcd }}^{\text {Large } N}=\frac{\rho_{n l}}{16 F^{2}}\left(\frac{N}{2 F^{2}}\right)^{n-1} \operatorname{tr}\left(\left[t^{a}, t^{b}\right]\left[t^{c}, t^{d}\right]\right), \quad l \quad \text { нечетное }
$$

где коэффициенты $\rho_{n l}$ удовлетворяют простому рекуррентному уравнению

$$
\rho_{n l}=\frac{1}{2(n-1)} \sum_{i=1}^{n-1} \sum_{l^{\prime}=0}^{n} \frac{\rho_{i l^{\prime}} \rho_{n-i, l^{\prime}}}{2 l^{\prime}+1}\left(\delta^{l^{\prime} l}+\Omega_{n}^{l^{\prime} l}\right)
$$

с начальным условием $\rho_{10}=\rho_{11}=1$. Выражение для четных $l$ достаточно сложное, и мы не будем его приводить. Его нетрудно получить из уравнения (13) с помощью соотношений (8). В любом случае амплитуда для четных $l$ выражается в терминах тех же коэффициентов $\rho_{n l}(14)$.

Итак, мы свели решение для $S U(\infty)$-модели главного кирального поля при произвольном числе размерностей в приближении ГЛ к решению очень простого рекуррентного уравнения (14). Это замечательное уравнение, оно обладает богатыми симметриями. K сожалению, мы пока не нашли его аналитического решения ${ }^{4}$, однако это уравнение можно решить численно до практически неограниченного петлевого порядка. Решение требует отдельного исследования.

\section{5. ЗАКЛЮЧЕНИЕ И ПЕРСПЕКТИВЫ}

Получено нелинейное рекуррентное соотношение (6) для коэффициентов ряда ГЛ в четырехмерной сигма-модели с полями на произвольном римановом многообразии. При дополнении начальными условиями (5) это уравнение позволяет получить данные коэффициенты в терминах геометрических характеристик риманова многообразия. Явно вычислены двухпетлевые ГЛ для произвольной сигма-модели. Мы допускаем, что уравнение (6) можно получить как некоторое уравнение движения для нелокального объекта в римановом многообразии. Если это удастся сделать, то коэффициент ряда ГЛ можно будет вывести с помощью разложения этого объекта по его нелокальности.

Если применить уравнение (6) к четырехмерной $S U(N)$-модели главного кирального поля, то это позволит свести решение этой теории при $N \rightarrow \infty$ и в приближении ГЛ к решению простого и симпатичного уравнения (14). Мы надеемся, что это уравнение удастся решить аналитически, используя богатые симметрии, которыми оно обладает. Такое решение может снабдить нас информацией о спектре $S U(\infty)$-модели главного кирального поля в случае произвольного числа пространственно-временных измерений.

\footnotetext{
4) Отметим, что уравнение (14) без члена, пропорционального $\Omega_{n}^{l^{\prime} l}$, имеет очень простое решение $\rho_{n l}=\delta_{l 0} / 2^{n-1}+\delta_{l 1} / 6^{n-1}$.
} 
Благодарности. Мы благодарны Дмитрию Дьяконову за то, что он воодушевил нас провести вычисления, представленные в настоящей работе. Нам очень помогли прояснившие многие моменты обсуждения с Дмитрием Дьяконовым, Колей Кивелем, Юлией Кошински и Виктором Петровым. Работа выполнена при частичной поддержке German Ministry of Education and Research (грант № 06BO9012) и Российской федеральной программы "Научно-педагогический потенциал инновационной России" (контракт 02.740.11.5154).

\section{Список литературы}

[1] А.Н. Васильев, Квантовополевая ренормгруппа в теории критического поведения и стохастической динамике, Изд-во ПИЯФ, СПб., 1998.

[2] S. Weinberg, Effective field theory, past and future, arXiv: 0908.1964.

[3] M. Büchler, G. Colangelo, Eur. Phys. J. C, 32:3 (2003), 427-442, arXiv: hep-ph/0309049.

[4] J. Bijnens, L. Carloni, Nucl. Phys. B, 827:1-2 (2010), 237-255, arXiv: 0909.5086; 843:1 (2011), 55-83, arXiv: 1008.3499.

[5] M. Bissegger, A. Fuhrer, Phys. Lett. B, 646:1-2 (2007), 72-79, arXiv: hep-ph/0612096.

[6] N. Kivel, M. V. Polyakov, A. Vladimirov, Phys. Rev. Lett., 101 (2008), 262001; arXiv: 0809.3236.

[7] N. A. Kivel, M. V. Polyakov, A. A. Vladimirov, Писъма в ЖКЭТФ, 89:11 (2009), 621-635; arXiv: 0904.3008.

[8] J. Koschinski, M. V. Polyakov, A. A. Vladimirov, Phys. Rev. D, 82 (2010), 014014; arXiv: 1004.2197.

[9] D. H. Friedan, Ann. Phys., 163:2 (1985), 318-419.

[10] J. Honerkamp, Nucl. Phys. B, 36:1 (1972), 130-140.

[11] P. S. Howe, G. Papadopoulos, K. S. Stelle, Nucl. Phys. B, 296:1 (1988), 26-48.

[12] B. Riemann, Neu Nachrichten der Königlichen Gesellschaft von Wissenschaften zu Göttingen, 13 (1868), 133.

[13] А.Н. Васильев, Ю. М. Письмак, Ю.Р. Хонконен, ТМФ, 46:2 (1981), 157-171; 47:3 (1981), 291-306; 50:2 (1982), 195-206.

[14] А. М. Поляков, Калибровочные поля и струнъ, Изд-во УдГУ, Ижевск, 1999.

[15] A. A. Vladimirov, Infrared logarithms in effective field theories, $\mathrm{PhD}$ Thesis, Ruhr-University, Bochum, Germany, 2010. 